PROCEEDINGS OF THE AMERICAN MATHEMATICAL SOCIETY

Volume 126, Number 7, July 1998, Pages 1931-1940

S 0002-9939(98)04499-2

\title{
ON THE COHOMOLOGY OF REGULAR DIFFERENTIAL FORMS AND DUALIZING SHEAVES
}

\author{
REINHOLD HÜBL AND XIAOTAO SUN
}

(Communicated by Wolmer V. Vasconcelos)

\begin{abstract}
If $Y$ is a local Dedekind scheme and $X / Y$ is a projective CohenMacaulay variety of relative dimension 1 , then $R^{1} f_{*} \omega_{X / Y}^{1}$ is torsionfree if and only if $X / Y$ is arithmetically Cohen-Macaulay for a suitable embedding in $\mathbb{P}_{k}^{n}$. If $X$ is regular then $R^{1} f_{*} \omega_{X / Y}^{1}$ is torsionfree whenever the multiplicity of the special fibre is not a multiple of the characteristic of the residue class field.
\end{abstract}

Regular differential forms play an important role in the local and global duality theory of varieties and morphisms, and have been studied extensively over the last few years (cf. [12], [5]). Given a complex projective manifold $X$ and a morphism $f: X \rightarrow Y$ to a reduced variety $Y$, J. Kollar has shown that $R^{i} f_{*} \omega_{X}$ is torsionfree for any $i \in \mathbb{N}$ (cf. [10]). Little is known in the case of mixed characteristics, though in particular in the case of arithmetic varieties the torsionfreeness of the cohomology of canonical sheaves is of great interest. It appears in Bloch's work on the de Rham discriminant [2], and the second author has shown that it allows one to control the ramification of the base change of arithmetic surfaces, hence giving explicit formulas and estimates for the self-intersection number of canonical sheaves (cf. [14]).

In this note we develop some general criteria which reduce the problem of torsionfreeness of the cohomology of canonical sheaves to a question on homogeneous coordinate rings. Using the special situation in the case of arithmetic surfaces, we are able to settle the question in the case that the multiplicity of the special fibre is not a multiple of the characteristic of the residue class field.

The work was done while the second author was a visiting fellow at the ICTP. He would like to express his thanks to the mathematics section of the ICTP for its hospitality. Both authors are grateful to Professor M. S. Narasimhan for helpful discussions.

\section{$\S 1$. Regular Differential FORMS AND RESiduAl COMPlEXeS}

Let $Y$ be an integral noetherian scheme and let $f: X \rightarrow Y$ be a projective morphism, Cohen-Macaulay, equi-dimensional of relative dimension $d$ and with geometrically connected fibres. We assume that $X$ is reduced and $f$ is generically smooth, i.e. that the extension $K(X) / K(Y)$ of meromorphic functions is a direct

Received by the editors December 18, 1996.

1991 Mathematics Subject Classification. Primary 13N05, 14F10.

The first author was partially supported by a Heisenberg-Stipendium of the Deutsche Forschungsgemeinschaft. 
product of separable field extensions. In this situation the sheaf $\omega_{X / Y}^{d}$ of relative regular differential forms of $X / Y$ is well defined (cf. [11]), and it comes equipped with an intergral

$$
\int_{X / Y}: R^{d} f_{*} \omega_{X / Y}^{d} \longrightarrow \mathcal{O}_{Y}
$$

such that $\left(\omega_{X / Y}^{d}, \int_{X / Y}\right)$ is a dualizing pair for $f$, i.e. $\omega_{X / Y}^{d}$ is quasi-isomorphic to Grothendieck's $f^{!} \mathcal{O}_{Y}$ (cf. [9]) in the derived category. In order to study the torsion of the higher direct images $R^{i} f_{*} \omega_{X / Y}^{d}$ we may assume that $Y=\operatorname{Spec}(D)$ for some complete discrete valuation ring $(D, \pi)$ whose residue class field $k=D / \pi D$ is algebraically closed. Write $X=\operatorname{Proj}(S)$ for some graded reduced $D$-algebra $S=$ $\bigoplus S_{n}$. By $\omega_{S / D}^{d+1}$ we denote the module of regular differential forms of $S / D$, and by $\mathcal{A}_{S}$ we denote the system of denominators consisting of those sequences $F_{1}, \ldots, F_{l}$ of homogeneous elements whose residue classes modulo $\pi$ form an $S / \pi S$-active sequence. For details on systems of denominators, modules $\mathcal{C}_{\mathcal{A}_{S}}^{p}(M)$ of generalized fractions of $M$ with respect to $\mathcal{A}_{S}$ and (unaugmented) Cousin-complexes $\mathcal{C}_{\mathcal{A}_{S}}(M)$ we refer the reader to [8] and [16], and also to [4]. Furthermore let $E$ be a $D$ injective hull of $k$ and set $\mathcal{E}:=\widetilde{E}$, the sheaf on $Y$ associated to $E$. Furthermore let $\mathcal{K}_{S}^{\bullet}(\mathfrak{m}):=\left(\mathcal{C}_{\mathcal{A}_{S}}^{\bullet}\left(\omega_{S / D}^{d+1} \otimes_{D} E\right), d^{\bullet}\right)[d+1]$ be the Cousin complex of $\omega_{S / D}^{d+1} \otimes_{D} E$ with respect to $\mathcal{A}_{S}$, and set $\mathcal{K}_{X}^{\bullet}(\mathfrak{m}):=\mathcal{K}_{S}^{\bullet}(\mathfrak{m})[-1]^{\sim}$.

1.1 Proposition. $\mathcal{C}_{\mathcal{A}_{S}}\left(S(l) \otimes_{D} E\right)^{\sim}$ is a flasque resolution of $f^{*} \mathcal{E}(l)$, and $\mathcal{K}_{X}^{\bullet}(\mathfrak{m})$ is a complex of injective $\mathcal{O}_{X}$-modules and a representative of $f^{!} \mathcal{E}$.

Proof. First note that $\mathcal{C}_{\mathcal{A}_{S}}(M)$ is exact and a resolution of $M$ if and only if $\mathcal{A}_{S}$ consists of poor $M$-regular sequences. Thus $\mathcal{C}_{\mathcal{A}_{S}}\left(S \otimes_{D} E\right)$ is in general not exact; however, for any homogeneous element $G \in S_{+}$of positive degree, $S_{G} / D$ is a CohenMacaulay-algebra, and hence all sequences $(\mathbf{F}) \in \mathcal{A}_{S}$ are $E \otimes_{D} S_{G}$-poor sequences (cf. [4], §1), implying that $\mathcal{C}_{\mathcal{A}_{S}}\left(S(l) \otimes_{D} E\right)^{\sim}$ is a resolution of $\left(S(l) \otimes_{D} E\right)^{\sim}=f^{*} \mathcal{E}$. As

$$
\mathcal{C}_{\mathcal{A}_{S}}^{p}\left(S \otimes_{D} E\right) \cong \bigoplus \mathcal{C}_{\mathcal{A}_{S}}^{p}(S \otimes E)_{\mathfrak{P}^{h}},
$$

where the sum runs over all homogeneous primes of $S / \pi S$ of height $p$, it follows that $\mathcal{C}_{\mathcal{A}_{S}}\left(S \otimes_{D} E\right)^{\sim}$ also is flasque. Similarly, for any homogeneous $G \in S_{+}$of positive degree, $\left(\omega_{S / D}^{d+1}\right)_{G}$ is a Gorenstein module, implying that $\left(\mathcal{C}_{\mathcal{A}_{S}}\left(\omega_{S / D}^{d+1} \otimes_{D} E\right)\right)_{G}$ is a graded injective resolution of $\omega_{S_{G} / D}^{d+1} \otimes E$; hence $\mathcal{K}_{X}^{\bullet}(\mathfrak{m})$ is an injective resolution of $\left(\omega_{S / R}^{d+1} \otimes_{D} E\right)^{\sim}=\left(\omega_{S / D}^{d+1}\right)^{\sim} \otimes_{\mathcal{O}_{X}} f^{*} \mathcal{E}$. As $\omega_{X / Y}^{d}=\left(\omega_{S / D}^{d+1}\right) \sim$ by [5], (2.6), the proposition follows.

1.2 Corollary. For $i<d$ we have

$$
H^{i}\left(X, f^{*} \mathcal{E}(l)\right)=H^{i}\left(\mathcal{C}_{\mathcal{A}_{S}}\left(S \otimes_{D} E\right)\right)_{l}
$$

and for $i=d$ we have

$$
H^{d}\left(X, f^{*} \mathcal{E}(l)\right)=\mathcal{C}_{\mathcal{A}_{S}}^{d+1}\left(S \otimes_{D} E\right)_{l} .
$$

Proof. First note that for $i \leq d$ we have

$$
\Gamma\left(X, \bigoplus_{l \in \mathbb{Z}} \mathcal{C}_{\mathcal{A}_{S}}^{i}\left(S(l) \otimes_{D} E\right)^{\sim}\right)=\mathcal{C}_{\mathcal{A}_{S}}^{i}\left(S \otimes_{D} E\right)
$$


as graded modules, and for $i=d+1$ we have

$$
\Gamma\left(X, \bigoplus_{l \in \mathbb{Z}} \mathcal{C}_{\mathcal{A}_{S}}^{d+1}\left(S(l) \otimes_{D} E\right) \sim()=0 .\right.
$$

Finally, for $(\mathbf{F}) \in \mathcal{A}_{S}^{d+1}$ or $(\mathbf{F}) \in \mathcal{A}_{S}^{d+2}$ we have that any $G \in S(\mathbf{F})$ is an $S /\left(\pi^{n}, \mathbf{F}\right) S_{-}$ regular element for trivial reasons $(n \in \mathbb{N})$, implying that

$$
\mathcal{C}_{\mathcal{A}_{S}}^{d-1}\left(S \otimes_{D} E\right) \longrightarrow \mathcal{C}_{\mathcal{A}_{S}}^{d}\left(S \otimes_{D} E\right) \longrightarrow \mathcal{C}_{\mathcal{A}_{S}}^{d+1}\left(S \otimes_{D} E\right) \longrightarrow 0
$$

is an exact sequence. From these facts the corollary follows immediately.

In this note we are interested in the torsionfreeness of $R^{i} f_{*} \omega_{X / Y}^{d}$. Clearly $f_{*} \omega_{X / Y}^{d}$ is torsionfree by the reducedness of $X$. For $i>0$ we have

1.3 Proposition. For $i>0$ the following conditions are equivalent:

i) The sheaf $R^{i} f_{*} \omega_{X / Y}^{d}$ is torsion free.

ii) The morphism

$$
\mu_{\pi}: \operatorname{Hom}_{\mathcal{O}_{Y}}\left(R^{i} f_{*} \omega_{X / Y}^{d}, \mathcal{E}\right) \rightarrow \operatorname{Hom}_{\mathcal{O}_{Y}}\left(R^{i} f_{*} \omega_{X / Y}^{d}, \mathcal{E}\right)
$$

is surjective.

iii) The morphism

$$
\mu_{\pi}: \operatorname{Hom}_{D}^{\text {cont }}\left(H_{S_{+}}^{i+1}\left(\omega_{S / D}^{d+1}\right), E\right)_{0} \rightarrow H_{D}^{c o n t}\left(H_{S_{+}}^{i+1}\left(\omega_{S / D}^{d+1}\right), E\right)_{0}
$$

is surjective.

iv) The map

$$
\mu_{\pi}: H^{-(i+1)}\left(\operatorname{Hom}_{S}\left(\omega_{S / D}^{d+1}, \mathcal{K}_{S}^{\bullet}(\mathfrak{m})\right)\right)_{0} \rightarrow H^{-(i+1)}\left(\operatorname{Hom}_{S}\left(\omega_{S / D}^{d+1}, \mathcal{K}_{S}^{\bullet}(\mathfrak{m})\right)\right)_{0}
$$
is surjective.

v) The morphism

$$
\mu_{\pi}: H^{d-i}\left(\mathcal{C}_{\mathcal{A}_{S}}\left(S \otimes_{D} E\right)\right)_{0} \rightarrow H^{d-i}\left(\mathcal{C}_{\mathcal{A}_{S}}\left(S \otimes_{D} E\right)\right)_{0}
$$

is surjective.

Proof. The equivalence of i) and ii) is an immediate consequence of the main theorem on finitely generated modules over principal ideal domains. ii) and iii) are equivalent by [5], (2.6). For $l<0$ we have that

$$
\operatorname{Hom}_{S}\left(\omega_{S / D}^{d+1}, \mathcal{K}_{S}^{l}(\mathfrak{m})\right)_{0} \cong \Gamma\left(X, \operatorname{Hom}_{\mathcal{O}_{X}}\left(\omega_{X / Y}^{d}, \mathcal{K}_{X}^{l-1}(\mathfrak{m})\right)\right),
$$

implying that

$$
\begin{aligned}
\operatorname{Ext}_{\mathcal{O}_{X}}^{d-i}\left(\omega_{X / Y}^{d}, \omega_{X / Y}^{d} \otimes \mathcal{E}\right) & \cong \operatorname{Ext}_{\mathcal{O}_{X}}^{-i}\left(\omega_{X / Y}^{d}, \mathcal{K}_{X}^{\bullet}(\mathfrak{m})\right) \\
& \cong H^{-(i+1)}\left(\operatorname{Hom}_{S}\left(\omega_{S / D}^{d+1}, \mathcal{K}_{S}^{\bullet}(\mathfrak{m})\right)\right)_{0}
\end{aligned}
$$

and the equivalence of ii) and iv) follows from [5], (4.2). Finally for the equivalence of ii) and v) we note that in the derived category

$$
f^{*} \tilde{N} \cong \operatorname{Hom}_{\mathcal{O}_{X}}\left(\omega_{X / Y}^{d}, \omega_{X / Y}^{d} \otimes \widetilde{N}\right)
$$

for any $D$-module $N$, implying that

$$
\operatorname{Hom}_{D}\left(R^{i} f_{*} \omega_{X / Y}^{d}, \mathcal{E}\right) \cong \operatorname{Ext}_{\mathcal{O}_{X}}^{d-i}\left(\mathcal{O}_{X}, \mathcal{O}_{X} \otimes \mathcal{E}\right) \cong H^{d-i}\left(\mathcal{C}_{\mathcal{A}_{S}}\left(S \otimes_{D} E\right)\right)_{0}
$$

by full duality for $X / Y$. 
1.4 Corollary. Suppose $X / Y$ is arithmetically $S_{k+2}$, i.e. $S$ and $S / \pi S$ satisy Serre's condition $S_{k+2}$. Then $R^{d-i} f_{*} \omega_{X / Y}^{d}$ is torsionfree and commutes with base change for all $i \leq k$.

Proof. As $S / D$ is flat and $S / \pi S$ is $S_{k+2}$, we get that $H^{d-i}\left(\mathcal{C}_{\mathcal{A}_{S}}(S \otimes E)\right)=0$ for $d-i \leq k$ by [17], (3.3) and [4], §1, and the claim follows from (1.3).

1.5 Corollary. Suppose that $X / Y$ is globally a complete intersection. Then the sheaf $R^{i} f_{*} \omega_{X / Y}^{d}$ is torsionfree as an $\mathcal{O}_{Y}$-module for all $i \in \mathbb{N}$.

1.6 Theorem. For $k<d$ the following conditions are equivalent:

i) The sheaf $R^{d-i} f_{*} \omega_{X / Y}^{d}$ is torsionfree for all $i \leq k$.

ii) $R^{i} f_{*} \mathcal{O}_{X}$ commutes with base change for $i \leq k$.

iii) $H^{i}\left(\mathcal{C}_{\mathcal{A}_{S}}\left(S \otimes_{D} E\right)\right)_{0} \cong H^{i}\left(X, \mathcal{O}_{X}\right) \otimes_{D} E$ for $i \leq k$.

iv) There is an $l>0$ such that

$$
H^{0}\left(\mathcal{C}_{\mathcal{A}_{S}}\left(S^{(l)} \otimes_{D} E\right)\right)=S^{(l)} \otimes E
$$

and

$$
H^{i}\left(\mathcal{C}_{\mathcal{A}_{S}}\left(S^{(l)} \otimes_{D} E\right)\right)=H^{i}\left(X, \mathcal{O}_{X}\right) \otimes_{D} E \quad \text { for } 1 \leq i \leq k .
$$

Proof. As full duality holds for $X / Y$, we may assume (after replacing $S$ by $S^{(l)}$ for some $l>0$ if necessary) that

$R^{d} f_{*} \mathcal{O}_{X}(p)$ is locally free for $p \neq 0$,

$R^{i} f_{*} \mathcal{O}_{X}(p)=0$ for $p \neq 0$ and $1 \leq i \leq d-1$,

$f_{*} \mathcal{O}_{X}(p)=\widetilde{S_{p}}$ for $p \neq 0$

(cf. [9], (11)). This implies the equivalence of iii) and iv) in view of (1.1) and the fact that $f_{*} \mathcal{O}_{X}=\mathcal{O}_{Y}$. Note, in particular, that the direct image sheaf $f_{*} \mathcal{O}_{X}(p)$ commutes with base change for any $p \neq 0$.

Assuming ii), we conclude from (1.2) that

$$
H^{i}\left(X, \mathcal{O}_{X}\right) \otimes_{D} E \cong H^{i}\left(X, f^{*} \mathcal{E}\right) \cong H^{i}\left(\mathcal{C}_{\mathcal{A}_{S}}\left(S \otimes_{D} E\right)\right)_{0} .
$$

Hence ii) implies iii), and iii) clearly implies i) by (1.3)v). Thus it remains to show that i) implies ii). As $R^{d} f_{*} \omega_{X / Y}^{d}$ commutes with base change, it follows from the assumptions that $R^{d-i} f_{*} \omega_{X / Y}^{d}$ is locally free and $R^{d-i-1} f_{*} \omega_{X / Y}^{d}$ commutes with base change for all $i \leq k$ (cf. [3], III. (12.11)). Hence for any $D$-module $N$ we have isomorphisms

$$
\begin{aligned}
H^{i}\left(X, f^{*} \widetilde{N}\right) \cong & \operatorname{Ext}_{\mathcal{O}_{X}}^{i}\left(\mathcal{O}_{X}, f^{*} \widetilde{N}\right) \\
& \cong \operatorname{Ext}_{\mathcal{O}_{X}}^{i}\left(\omega_{X / Y}^{d}, \omega_{X / Y}^{d} \otimes f^{*} \widetilde{N}\right) \\
& \cong \operatorname{Hom}_{D}\left(H^{d-i}\left(X, \omega_{X / Y}^{d}\right), N\right) \\
& \cong \operatorname{Hom}_{D}\left(H^{d-i}\left(X, \omega_{X / Y}^{d}\right), D\right) \otimes_{D} N \\
& \cong \operatorname{Ext}_{\mathcal{O}_{X}}^{i}\left(\omega_{X / Y}^{d}, \omega_{X / Y}^{d}\right) \otimes N \\
& \cong H^{i}\left(X, \mathcal{O}_{X}\right) \otimes_{D} N
\end{aligned}
$$

by full duality for $X / Y$ (cf. [9], (10) ii) b).

In general, the sheaves $R^{i} f_{*} \mathcal{O}_{X}$ are much harder to study than the sheaves $R^{i} f_{*} \omega_{X / Y}^{d}$ (cf. [10]). However, by the above we get in view of base change theory ([3], III. (12.11)) 
1.7 Corollary. Suppose that $R^{d-i} f_{*} \omega_{X / Y}^{d}$ is torsionfree for all $i \leq k$. Then $R^{i} f_{*} \mathcal{O}_{X}$ is torsionfree for all $i \leq k$.

1.8 Corollary. Suppose $d=1$, i.e. $X$ is a surface. Then $R^{1} f_{*} \omega_{X / Y}^{1}$ is torsionfree if and only if there exists a graded Cohen-Macaulay algebra $S / D$ such that $X=$ $\operatorname{Proj}(S)$.

Proof. If $S / D$ is Cohen-Macaulay, then $R^{1} f_{*} \omega_{X / Y}^{1}$ is torsionfree by (1.4). For the converse we may assume that $S$ is normal. As we have seen in the proof of (1.1), we already have an exact sequence

$$
\mathcal{C}_{\mathcal{A}_{S}}^{0}\left(S \otimes_{D} E\right) \longrightarrow \mathcal{C}_{\mathcal{A}_{S}}^{1}\left(S \otimes_{D} E\right) \longrightarrow \mathcal{C}_{\mathcal{A}_{S}}^{2}\left(S \otimes_{D} E\right) \longrightarrow 0,
$$

and by (1.6) we also may assume that

$$
H^{0}\left(\mathcal{C}_{\mathcal{A}_{S}}\left(S \otimes_{D} E\right)\right) \cong S \otimes_{D} E,
$$

so that $\mathcal{C}_{\mathcal{A}_{S}}\left(S \otimes_{D} E\right)$ is a resolution of $S \otimes_{D} E$. Thus all sequences in $\mathcal{A}_{S}$ are poor $S \otimes_{D} E$-sequences by [17], (3.3). Any homogeneous $S \otimes_{D} E$-regular sequence $\left\{F_{1}, F_{2}\right\}$ in $S_{+}$is also an $S / \pi S=\operatorname{Hom}_{S}(S / \pi S, S \otimes E)$-regular sequence by the graded version of [7], (E.9). Hence $S / \pi S$ is Cohen-Macaulay, and the corollary follows.

\section{§2. CANONical Sheaves on ARithmetic SURfaces}

Much more special than the general case is the case of arithmetic surfaces, i.e. we assume that $D$ is a complete discrete valuation ring whose residue class field $k$ is algebraically closed with $\operatorname{char}(k)=p \geq 0$, $\operatorname{char}(Q(D))=0, Y=\operatorname{Spec}(D)$, that $X$ is a regular scheme of dimension 2, and that $f: X \rightarrow Y$ is proper and flat with connected fibres. By $\eta$ resp. $y$ we denote the generic resp. the closed point of $Y$, and by $X_{\eta}$ resp. $X_{y}$ the generic resp. the special fibre of $f$. Write $X_{y}=\sum_{i=1}^{m} n_{i} F_{i}$ with reduced and irreducible curves $F_{i}$, and set $n=$ g.c.d. $\left(n_{1}, \ldots, n_{m}\right), k_{i}=\frac{n_{i}}{n}$ and $F:=\sum_{i=1}^{m} k_{i} F_{i}$.

2.1 Lemma. $\mathcal{L}:=\mathcal{O}_{X}(F)$ is a torsion element of Pic $(X)$ of order exactly $n$.

Proof. Let $\pi$ be the local parameter of $D$. Then

$$
\mathcal{L}^{\otimes n}=\mathcal{O}_{X}(n F)=\mathcal{O}_{X} \pi^{-1} \cong \mathcal{O}_{X},
$$

so that $\mathcal{L}$ is torsion and $r=\operatorname{ord}(\mathcal{L}) \mid n$. Furthermore $\mathcal{L}^{\otimes r}=\mathcal{O}_{X}(r F) \cong \mathcal{O}_{X}$, so that there exists an $s \in H^{0}\left(X, \mathcal{L}^{\otimes r}\right)=H^{0}\left(X, \mathcal{O}_{X}\right)=D$ such that $(s)_{0}=r F$. But obviously

$$
(s)_{0}\left\{\begin{array}{cc}
=0 & \text { if } \pi \nmid s, \\
\geq(t)_{0} & \text { if } \pi \mid s,
\end{array}\right.
$$

implying that $r=0$ or $r \geq n$.

2.2 Lemma. Let $\mathcal{L}$ be an invertible sheaf on $F$ with $\operatorname{deg}\left(\mathcal{L} \mid F_{i}\right)=0$ for $i \in$ $\{1, \ldots, m\}$. Then $h^{0}(F, \mathcal{L}) \leq 1$, and $h^{0}(F, \mathcal{L})=1$ if and only if $\mathcal{L}=\mathcal{O}_{F}$. 
Proof. Assume that $h^{0}(F, \mathcal{L}) \geq 1$ and let $s \in H^{0}(F, \mathcal{L}) \backslash\{0\}$ be a section. Let $D_{1}=\sum r_{i} F_{i}$ be the maximal subdivisor of $F$ with $s \mid D_{1}=0$, and write $F=D_{1}+D_{2}$. Hence $s \in H^{0}\left(F, \mathcal{O}_{D_{2}}\left(-D_{1}\right) \otimes \mathcal{L}\right)$, and $s$ induces an exact sequence

$$
0 \longrightarrow \mathcal{O}_{D_{2}} \longrightarrow \mathcal{O}_{D_{2}}\left(-D_{1}\right) \otimes \mathcal{L} \longrightarrow \mathcal{C} \longrightarrow 0,
$$

where $\mathcal{C}$ is a sheaf on $F$ with zero-dimensional support (by the maximality of $D_{1}$ ). The Riemann-Roch theorem on $D_{2}$ implies

$$
-D_{1} \cdot D_{2}=\chi\left(\mathcal{O}_{D_{2}}\left(-D_{1}\right) \otimes \mathcal{L}\right)-\chi\left(\mathcal{O}_{D_{2}}\right)=\chi(\mathcal{C})=h^{0}(F, \mathcal{L}) \geq 0 .
$$

Thus $D_{1}^{2}=D_{2}^{2}=-D_{1} \cdot D_{2} \geq 0$ (as $D_{i} \cdot F=0$ ), implying by [1], (1.1)d that there exist $\frac{p_{1}}{q_{1}}, \frac{p_{2}}{q_{2}} \in \mathbb{Q}$ such that $D_{1}=\frac{p_{1}}{q_{1}} X_{y}, D_{2}=\frac{p_{2}}{q_{2}} X_{y}$ with $q_{i} \mid n$. As $s \neq 0$ we have $D_{2} \neq 0$; hence $D_{1}<F$, i.e. $D_{1}=0, D_{2}=F$, and $s: \mathcal{O}_{F} \rightarrow \mathcal{L}$ is an isomorphism.

Now set $\mathcal{L}=\mathcal{O}_{X}(F)$ again. Then $\mathcal{L} \mid F=\mathcal{O}_{F}(F)$ is a torsion element of $\operatorname{Pic}(F)$.

2.3 Lemma. If $\operatorname{ord}(\mathcal{L} \mid F)=l$, then $h^{0}\left(F, \mathcal{O}_{\nu F}\right)=1$ for any $\nu \in\{1, \ldots, l\}$.

Proof. By $(2.2)$ we have $h^{0}\left(F, \mathcal{O}_{F}(-\nu F)\right)=0$ for $0<\nu<l$. Thus the exact sequence

$$
0 \longrightarrow \mathcal{O}_{F}(-\nu F) \longrightarrow \mathcal{O}_{(\nu+1) F} \longrightarrow \mathcal{O}_{\nu F} \longrightarrow 0
$$

implies $h^{0}\left(F, \mathcal{O}_{(\nu+1) F}\right) \leq h^{0}\left(F, \mathcal{O}_{\nu F}\right)$ for $\nu<l$. As $h^{0}\left(F, \mathcal{O}_{F}\right)=1$ (cf. [1], (2.6)), and as $h^{0}\left(F, \mathcal{O}_{F}(-l F)\right)=h^{0}\left(F, \mathcal{O}_{F}\right)=1$, the lemma follows.

2.4 Proposition. If $p \nmid n$, then the order of $\mathcal{L} \mid F$ is exactly $n$.

Proof. $\mu_{n}=\operatorname{Spec}\left(\mathbb{Z}[T] /\left(T^{n}-1\right)\right)$ is a finite group scheme over $\mathbb{Z}$. For any scheme $Z$ we write $\mu_{n, Z}=Z \times_{\text {Spec } \mathbb{Z}} \mu_{n}$, and we denote by $Z_{f l}$ resp. $Z_{e t}$ the scheme $Z$ equipped with the flat resp. étale topology. Then we have a commutative diagram

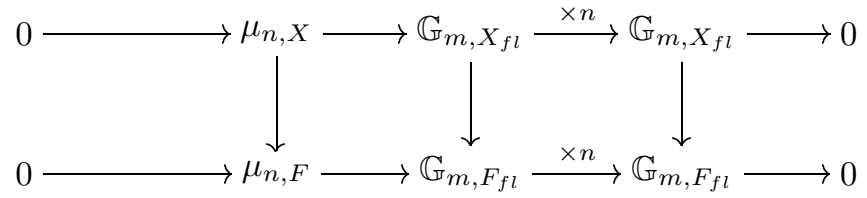

with exact rows. As $H_{f l}^{0}\left(F, \mathbb{G}_{m, F_{f l}}\right)=k^{*}$,

$$
H_{f l}^{1}\left(F, \mathbb{G}_{m, F_{f l}}\right)=\operatorname{Pic}(F) \quad \text { resp. } H_{f l}^{1}\left(X, \mathbb{G}_{m, X_{f l}}\right)=\operatorname{Pic}(X)
$$

(cf. [13], III.(3.9)), and as $k$ is algebraically closed we obtain a commutative diagram

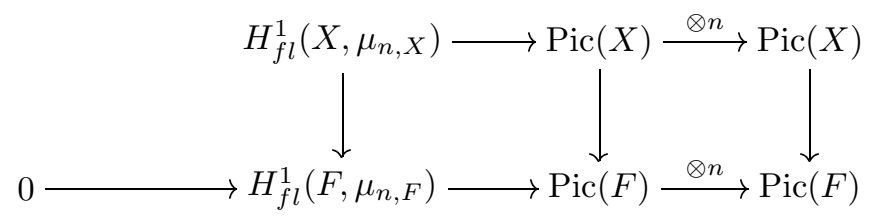

with exact rows. If $p \nmid n$, then $\mu_{n, X}$ and $\mu_{n, F}$ are étale group schemes over $X$ resp. $F$; hence their flat and étale cohomology coincide. By the proper base change theorem for étale cohomology this implies that $H_{f l}^{1}\left(X, \mu_{n, X}\right) \rightarrow H_{f l}^{1}\left(F, \mu_{n, F}\right)$ is an isomorphism (as $X_{y} \cong F$ as topological spaces). $\operatorname{Thus} \operatorname{ord}(\mathcal{L} \mid F)=\operatorname{ord}(\mathcal{L})=n$.

2.5 Theorem. In the above situation assume that $p \nmid n$. Then $h^{0}\left(X, \mathcal{O}_{X_{y}}\right)=1$, and $R^{1} f_{*} \omega_{X / Y}^{1}, R^{1} f_{*} \mathcal{O}_{X}$ are torsionfree. 
Proof. By (2.4) and (2.3) we get $h^{0}\left(X, \mathcal{O}_{X_{y}}\right)=1$; hence $h^{0}\left(X, \mathcal{O}_{X_{\eta}}\right)=h^{0}\left(X, \mathcal{O}_{X_{y}}\right)$ $=1$, and we conclude that $f_{*} \mathcal{O}_{X}$ commutes with base change, implying by (1.6) that $R^{1} f_{*} \omega_{X / Y}^{1}$ is torsionfree and by base change theory that $R^{1} f_{*} \mathcal{O}_{X}$ is torsionfree.

2.6 Corollary. In the situation of (2.5) assume that $X / Y$ is projective. Then there exists a graded Cohen-Macaulay algebra $S / D$ such that $X=\operatorname{Proj}(S)$.

2.7 Conjecture. For any projective arithmetic surface $X / Y$ there exists a graded Cohen-Macaulay algebra $S / D$ such that $X=\operatorname{Proj}(S)$.

2.8 Remark. i) If $X$ is a regular scheme of $\operatorname{dim}(X)>2$, then $R^{i} f_{*} \omega_{X / Y}^{d}$ may be torsionfree for all $i \in \mathbb{N}$ without $X / Y$ being arithmetically Cohen-Macaulay.

ii) If $\operatorname{dim}(X)=2$ and $X / Y$ is projective and Cohen-Macaulay, then $X / Y$ need not be arithmetically Cohen-Macaulay, and in particular $R^{1} f_{*} \omega_{X / Y}^{1}$ need not be torsionfree (cf. [6]). Thus the above conjecture is the strongest result one might hope for.

iii) If $g$ is the genus of $X_{\eta}$ and $X_{y}=n \cdot F$, then $n(g(F)-1)=g-1$, and hence $R^{1} f_{*} \omega_{X / Y}^{1}$ is torsionfree if $p \nmid(g-1)$.

iv) If the canonical map $H_{f l}^{1}\left(X, \mu_{n, X}\right) \rightarrow H_{f l}^{1}\left(F, \mu_{n, F}\right)$ is injective for any $n \in \mathbb{N}$ (for instance if we have a proper base change theorem in flat cohomology), then the conclusion of $(2.5)$ holds without any assumptions on $(p, n)$.

\section{§3. Good base extensions}

Given an arithmetic surface $f: X \rightarrow Y$ as in section 2, the second author examined in [15] the effects of base extensions on the self-intersection number of the canonical divisor and the degree of its direct image. In this section we will examine the effects of base extension on the higher direct images $R^{1} f_{*} \omega_{X / Y}^{1}$ and $R^{1} f_{*} \mathcal{O}_{X}$. Again we assume that we are in the local case, i.e. $Y=\operatorname{Spec}(D)$ for some complete discrete valuation ring $D$ with algebraically closed residue class field. Assume that $\widetilde{D} / D$ is a finite extension of discrete valuation rings, set $\widetilde{Y}:=\operatorname{Spec}(\widetilde{D})$, and consider the following diagram:

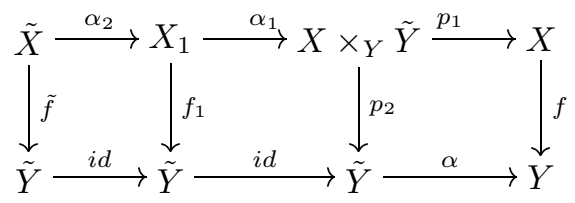

where $\alpha_{1}$ is the normalization of $X \times_{Y} \tilde{Y}$ and $\alpha_{2}$ is the minimal desingularization of $X_{1}$. Set $\Phi:=p_{1} \circ \alpha_{1}$ and $\varphi:=\Phi \circ \alpha_{2}$.

For any coherent sheaf $\mathcal{F}$ on $\widetilde{X}$ the Leray spectral sequence for composite functors gives exact sequences

$$
0 \longrightarrow R^{1} f_{*}\left(\varphi_{*} \mathcal{F}\right) \longrightarrow R^{1}(f \circ \varphi)_{*} \mathcal{F} \longrightarrow f_{*}\left(R^{1} \varphi_{*} \mathcal{F}\right) \longrightarrow 0
$$

and

$$
0 \longrightarrow R^{1} \alpha_{*}\left(\widetilde{f}_{*} \mathcal{F}\right) \longrightarrow R^{1}(\alpha \circ \widetilde{f})_{*} \mathcal{F} \longrightarrow \alpha_{*}\left(R^{1} \widetilde{f}_{*} \mathcal{F}\right)
$$

Since $f \circ \varphi=\alpha \circ \widetilde{f}$ and $R^{1} \alpha_{*}\left(\widetilde{f}_{*} \mathcal{F}\right)=0$, we get an inclusion

$$
R^{1} f_{*}\left(\varphi_{*} \mathcal{F}\right) \hookrightarrow \alpha_{*}\left(R^{1} \tilde{f}_{*} \mathcal{F}\right)
$$


In particular, for $\mathcal{F}=\mathcal{O}_{\widetilde{X}}$ we get $R^{1} f_{*}\left(\varphi_{*} \mathcal{O}_{\widetilde{X}}\right) \subseteq \alpha_{*}\left(R^{1} \widetilde{f}_{*} \mathcal{O}_{\widetilde{X}}\right)$, and we would like to know if we also have

$$
R^{1} f_{*} \mathcal{O}_{X} \hookrightarrow \alpha_{*}\left(R^{1} \widetilde{f}_{*} \mathcal{O}_{\widetilde{X}}\right)
$$

Definition. $\alpha$ is called a good base extension, if $(*)$ holds for $\alpha$.

3.1 Theorem. Let $F_{1}$ be the closed fibre of $f_{1}: X_{1} \rightarrow \tilde{Y}$ and $d=\operatorname{deg}(\alpha)$. If the conductor divisor $\mathfrak{C}\left(\alpha_{1}\right)$ of $\alpha_{1}$ satisfies $\mathfrak{C}\left(\alpha_{1}\right) \leq(d-1) F_{1}$, then $\alpha$ is a good base extension. In particular, $\alpha$ is a good base extension if $(p, d)=1$.

Proof. We may write $\widetilde{D}=D[[T]] /(f(T))$ for some polynomial $f(T)$ of degree $d$. Let $\operatorname{Tr}: K(\widetilde{X}) \rightarrow K(X)$ be the canonical trace. Then $\operatorname{Tr}\left(\frac{T^{d-1}}{f^{\prime}(T)}\right)=1$ by [7], app. F. On the other hand, by the proof of (2.1) of [15] we have $\mathfrak{C}\left(\alpha_{1}\right)=f_{1}^{*} R(\alpha)-R(\Phi)$, where $R(g)$ denotes the ramification divisor of a morphism $g$ (cf. [15]). We set $U=\left(X_{1}\right)_{\text {reg }}$, the regular locus of $X_{1}$, and we denote by $\mathcal{O}_{X_{1}}(R(\Phi))$ the unique extension of $\mathcal{O}_{U}(R(\Phi))$. Then for any codimension one point $x \in X_{1}$ we have

$$
\mathcal{O}_{X_{1}}(R(\Phi))_{x}=\mathcal{O}_{X_{1}, x} \cdot \gamma^{-1},
$$

where $\gamma$ is the local equation of $R(\Phi)=f_{1}^{*} R(\alpha)-\mathfrak{C}\left(\alpha_{1}\right)$ at $x$. If $v_{x}$ is the valuation on $K(\widetilde{X})$ determined by $x$, then $v_{x}(\gamma)=v_{x}\left(f^{\prime}(T)\right)-v_{x}(c)$, where $c$ is a local equation of $\mathfrak{C}\left(\alpha_{1}\right)$ at $x$. By our assumption on the conductor divisor,

$$
v_{x}(\gamma) \geq v_{x}\left(f^{\prime}(T)\right)-v_{x}\left(T^{d-1}\right)=v_{x}\left(\frac{f^{\prime}(T)}{T^{d-1}}\right),
$$

which implies that $\frac{T^{d-1}}{f^{\prime}(T)} \in \mathcal{O}_{X_{1}, x} \gamma^{-1}$. Thus $\frac{f^{\prime}(T)}{T^{d-1}}$ defines a global section of $\mathcal{O}_{X_{1}}(R(\Phi))$, as $X_{1}$ is normal. We obtain a morphism of $\mathcal{O}_{X^{-}}$modules

$$
\iota: \varphi_{*} \mathcal{O}_{X_{1}} \longrightarrow K(X), \quad g \longmapsto \iota(g):=\operatorname{Tr}\left(g \cdot \frac{f^{\prime}(T)}{T^{d-1}}\right),
$$

satisfying $\iota\left(\Phi_{*} \mathcal{O}_{X_{1}}\right) \subseteq \mathcal{O}_{X}$. It suffices to check this at points $x$ of codimension 1 . In this case $\mathcal{O}_{X_{1}, x} \cdot \gamma$ is the Kähler different of $\mathcal{O}_{X_{1}, x} / \mathcal{O}_{X, \varphi(x)}$, which coincides with the Dedekind different in our situation ([7], (10.17)), and we obtain

$$
\mathcal{O}_{X_{1}}(R(\Phi))=\mathcal{O}_{X_{1}, x} \cdot \gamma^{-1}=\left\{h \in K(\widetilde{X}): \operatorname{Tr}\left(h \cdot \mathcal{O}_{X_{1}, x}\right) \subseteq \mathcal{O}_{X, \varphi(x)}\right\} ;
$$

and as $\frac{f^{\prime}(T)}{T^{d-1}} \in \mathcal{O}_{X_{1}}(R(\Phi))_{x}$, this implies $\iota\left(\Phi_{*}\left(\mathcal{O}_{X_{1}}\right)\right) \subseteq \mathcal{O}_{X}$. Hence we get a morphism $\iota: \Phi_{*} \mathcal{O}_{X_{1}} \rightarrow \mathcal{O}_{X}$ which splits the canonical inclusion $\mathcal{O}_{X} \rightarrow \Phi_{*} \mathcal{O}_{X_{1}}$, and we get

$$
\Phi_{*} \mathcal{O}_{X_{1}} \cong \mathcal{O}_{X} \oplus \mathcal{E}
$$

As $\varphi_{*} \mathcal{O}_{\widetilde{X}}=\Phi_{*} \mathcal{O}_{X_{1}}$, this implies

$$
R^{1} f_{*} \mathcal{O}_{X} \hookrightarrow R^{1} f_{*}\left(\varphi_{*} \mathcal{O}_{\widetilde{X}}\right) \hookrightarrow \alpha_{*}\left(R^{1} \widetilde{f}_{*} \mathcal{O}_{\widetilde{X}}\right),
$$

and $\alpha$ is a good base extension. If $(p, d)=1$ then $f_{1}^{*} R(\alpha)=(d-1) F_{1}$ by [15], and the condition $\mathfrak{C}\left(\alpha_{1}\right) \leq(d-1) F_{1}$ is satisfied.

We say that $f: X \rightarrow Y$ has no multiple fibre if the special fibre $X_{y}=\sum_{i=1}^{m} n_{i} F_{i}$ satisfies $\operatorname{gcd}\left(n_{1}, \ldots, n_{m}\right)=1$. 
3.2 Proposition. Let $\pi$ be a parameter of $D$; write $X_{y}=n \cdot F, \widetilde{D}=D[T] /\left(T^{n}-\pi\right)$ and $\alpha: \widetilde{Y}:=\operatorname{Spec} \widetilde{D} \rightarrow Y$. Assume that $(p, n)=1$. Then in the above diagram $\widetilde{X}=X_{1}$, and $\varphi: X_{1} \rightarrow X$ is an étale cover of degree $n$. In particular, there exists a good base extension $\alpha: \widetilde{Y} \rightarrow Y$ such that $\tilde{f}: \widetilde{X} \rightarrow \widetilde{Y}$ has no multiple fibre.

Proof. Let $x_{i}$ be the generic point of an irreducible component $F_{i}$ of $X_{y}$, and write $\mathfrak{m}_{x}=u_{i} \mathcal{O}_{X, x_{i}}$. Then $\pi=a_{i} u^{n k_{i}}$ for some unit $a_{i} \in \mathcal{O}_{X, x_{i}}$, and hence

$$
K(\widetilde{X})=K(X)[T] /\left(T^{n}-a_{i} u_{i}^{n k_{i}}\right)=K(X)\left[T_{i}\right] /\left(T_{i}^{n}-a_{i}\right),
$$

where $T_{i}=\frac{T}{u^{k_{i}}}$. Thus $B:=\mathcal{O}_{X, x_{i}}\left[T_{i}\right] /\left(T_{i}^{n}-a_{i}\right)$ is étale over $\mathcal{O}_{X, x_{i}}$; hence it is the integral closure of $\mathcal{O}_{X, x_{i}}$ in $K(\widetilde{X})$. Thus $\Phi$ is unramified in codimension 1. From this we conclude that $R(\varphi)$ is exceptional, i.e. $\varphi(R(\varphi))$ is of codimension 2. As $\varphi^{*} \omega_{X / Y}^{1}-\omega_{\widetilde{X} / \widetilde{Y}}^{1}=\widetilde{f}^{*} R(\alpha)-R(\varphi)$ by [15], $(2.1)$, as $R(\varphi) \cdot \varphi^{*} \omega_{\widetilde{X} / \widetilde{Y}}^{1}=0$ and as $\alpha_{2}$ is a minimal desingularization we conclude that

$$
R(\varphi)^{2}=\omega_{\widetilde{X} / \widetilde{Y}}^{1} \cdot R(\varphi) \geq 0 .
$$

Thus $R(\varphi)=0$, i.e. $\widetilde{X}=X_{1}$, and $\varphi=\Phi$ is an étale cover of degree $n$. Obviously $\widetilde{f}: \widetilde{X} \rightarrow \widetilde{Y}$ has no multiple fibre.

As an easy consequence of (3.2) we obtain another proof of (2.3):

3.3 Corollary. If $(p, n)=1$, then $R^{1} f_{*} \mathcal{O}_{X}$ and $R^{1} f_{*} \omega_{X / Y}^{1}$ are torsionfree.

Proof. By (3.2) there exists a good base extension $\widetilde{Y} \rightarrow Y$ such that $\widetilde{f}: \widetilde{X} \rightarrow \widetilde{Y}$ has no multiple fibre. By [1], (2.6) this implies that $h^{0}\left(X, \mathcal{O}_{\widetilde{X}_{\tilde{y}}}\right)=1$, hence that $R^{1} \widetilde{f}_{*} \mathcal{O}_{\widetilde{X}}$ and $R^{1} \widetilde{f}_{*} \omega_{\widetilde{X} / \widetilde{Y}}^{1}$ are torsionfree, and the claim follows.

3.4 Remark. i) In the proof of (3.2) we wrote $\pi=a_{i} u_{i}^{n k_{i}}$ in the local ring $\mathcal{O}_{X, x_{i}}$ of $F_{i}$. If $\overline{a_{i}} \notin k\left(x_{i}\right)^{p}$ for all $i=1, \ldots, m$, then one can show by direct calculation that $\widetilde{Y} \rightarrow Y$ is a good base extension and $\tilde{f}: \widetilde{X} \rightarrow \widetilde{Y}$ has no multiple fibre (with no assumption on $(p, n))$. In particular, $R^{1} f_{*} \omega_{X / Y}^{1}$ and $R^{1} f_{*} \mathcal{O}_{X}$ are torsionfree in this case.

ii) Let $g: Z \rightarrow Y$ be a normal proper surface, and let $\Psi: X \rightarrow Z$ be a $Y$ birational proper map. Then $\Psi_{*} \mathcal{O}_{X}=\mathcal{O}_{Z}$, and the Leray spectral sequence gives an exact sequence

$$
0 \longrightarrow R^{1} g_{*} \mathcal{O}_{Z} \longrightarrow R^{1} f_{*} \mathcal{O}_{X} \longrightarrow g_{*} R^{1} \Psi_{*} \mathcal{O}_{X} \longrightarrow 0
$$

Thus if one can find a normal surface $f: X \rightarrow Y$ such that $R^{1} f_{*} \omega_{X / Y}^{1}$ is not torsionfree, one also would get a counterexample to conjecture 2.7 .

\section{REFERENCES}

[1] Artin, M. and G. Winters: Degenerate fibres and stable reduction of curves. Topology 10 (1971), 373 - 383. MR 57:16313

[2] Bloch, S.: De Rham cohomology and conductors of curves. Duke Math. Jour. 54 (1987), 295 - 308. MR 89h:11028

[3] Hartshorne, R.: Algebraic Geometry. Springer, Berlin, Heidelberg, New York (1977). MR $\mathbf{5 7 : 3 1 1 6}$

[4] HÜBL R.: Graded duality and generalized fractions. preprint.

[5] HÜBL, R. AND E. KunZ: Regular differential forms and duality for projective morphisms. Jour. reine angew. Math. 410 (1990), 84 - 108. MR 92a:14015 
[6] HÜBL, R. AND X. Sun: Vector bundles on the projective line over a discrete valuation ring. In preparation.

[7] Kunz, E.: Kähler differentials. Vieweg, Braunschweig, Wiesbaden (1986). MR 88e:14025

[8] Kersken, M.: Cousinkomplexe und Nennersysteme. Math. Z. 182 (1983), 389 - 402. MR 85h:32014

[9] Kleiman, S.: Relative duality for quasi-coherent sheaves. Comp. Math. 41 (1980), 39 - 60. MR 81m: 14017

[10] Kollar, J.: Higher direct images of dualizing sheaves I. Annals of Math. 123 (1986), 11 42. MR 87c: 14038

[11] Kunz, E. AND R. Waldi: Regular differential forms. Contemporary Mathematics 79 (1988), Amer. Math. Soc., Providence. MR 90a:14021

[12] Lipman, J.: Dualizing sheaves, differentials and residues on algebraic varieties. Astérisque 117 (1984). MR 86g:14008

[13] Milne, J.: Étale Cohomology. Princeton University Press, Princeton (1980). MR 81j:14002

[14] Sun, X.: Ramifications on arithmetic schemes. Jour. reine angew. Math. 488 (1997), 37-54.

[15] Sun, X.: On relative canonical sheaves of arithmetic surfaces. Math. Z. 223 (1996), 709 723. MR 97k: 14022

[16] Sharp, R. And H. Zakeri: Modules of generalized fractions. Mathematika 29 (1982), 32-41.

[17] SHARP, R. AND H. ZAKERI: Modules of generalized fractions and balanced big CohenMacaulay modules. Commutative Algebra: Durham 1981. London Math. Soc. Lecture Notes 72, (1982), 61 - 82. MR 84j:13021

Fachbereich Mathematik, Universiät Regensburg, D - 93040 Regensburg, Germany

E-mail address: reinhold.huebl@mathematik.uni-regensburg.de

International Centre for Theoretical Physics, Mathematics Section, 34100 Trieste, ITALY

E-mail address: xsun@ictp.trieste.it

Current address: Institute of Mathematics, Academia Sinica, Beijing 1000 80, People's Republic of China 